\title{
STABLE SYMMETRIES OF PLANE SEXTICS
}

\author{
Alex Degtyarev
}

Abstract. We classify projective symmetries of irreducible plane sextics with simple singularities which are stable under equivariant deformations. We also outline a connection between order 2 stable symmetries and maximal trigonal curves.

\section{INTRODUCTION}

1.1. Motivation. In a recent series of papers [8], [9], [10], we described the moduli spaces and computed the fundamental groups of a number of plane sextics. A common feature of all these papers is the fact that we start with proving that each sextic in the equisingular stratum under consideration possesses a certain symmetry (projective automorphism); then, this symmetry is used to analyze the moduli space and to write down explicit equations defining each curve. (Note that, prior to the papers cited above, for most curves in question only the existence was known, which was proved by rather indirect means.) In most cases, the symmetry can also be used to facilitate the computation of the fundamental group: in a certain sense, one can reduce pencils of degree six to those of degree four.

Thus, a natural question arises: are there other plane sextic curves admitting a symmetry stable under equisingular deformations? In the present paper, we give a partial answer to this question: our principal result is Theorem 1.3.1, classifying all irreducible plane sextics with simple singularities whose group of stable symmetries is nontrivial. Our approach should also apply to reducible sextics with simple singularities, but the number of cases to be considered and the number of classes obtained should be much larger. On the other hand, it appears that sextics with a non-simple singular point do not admit stable symmetries, $c f$. Remark 4.2.4.

In a subsequent paper, we are planning to use the results obtained here to compute the fundamental groups of all sextics listed in Theorem 1.3.1.

1.2. Stable symmetries. The (combinatorial) set of singularities of a plane curve $B$ is denoted by $\Sigma=\Sigma_{B}$. If all singularities are simple, we identify $\Sigma$ with the lattice spanned by the classes of exceptional divisors in the minimal resolution of singularities of the double plane ramified at $B, c f 2.3$; for this reason, we use $\oplus$ in the notation.

For an integer $m \geqslant 1$, we denote by $\mathcal{C}_{m}$ the space of plane curves of degree $m$; as is well known, $\mathcal{C}_{m}$ is a projective space of dimension $n(n+3) / 2$. Given a set of singularities $\Sigma$, the equisingular stratum $\mathcal{C}_{m}(\Sigma) \subset \mathcal{C}_{m}$ is the set of curves whose set of singularities is $\Sigma$. (Throughout this paper, all singularities are isolated and, in

2000 Mathematics Subject Classification. Primary: 14H45; Secondary: 14H30.

Key words and phrases. Plane sextic, symmetry, torus type, trigonal curve. 
most cases, even simple.) There is an obvious action of $P G L(3, \mathbb{C})$ on $\mathcal{C}_{m}$ preserving each stratum $\mathcal{C}_{m}(\Sigma)$; the quotient $\mathcal{M}_{m}(\Sigma)=\mathcal{C}_{m}(\Sigma) / P G L(3, \mathbb{C})$ is called the moduli space of curves of degree $m$ with the set of singularities $\Sigma$.

1.2.1. Definition. A symmetry of a plane curve $B \subset \mathbb{P}^{2}$ is an automorphism of the pair $\left(\mathbb{P}^{2}, B\right)$. (We use the term 'symmetry' instead of 'automorphism' to avoid confusion with automorphisms of $B$ as abstract curve.) A symmetry $s$ of $B$ is called stable if there is a neighborhood $U$ of $B$ in the equisingular stratum $\mathcal{C}_{m}\left(\Sigma_{B}\right)$ and a continuous family $\sigma: U \rightarrow P G L(3, \mathbb{C})$ such that $\sigma\left(B^{\prime}\right)$ is a symmetry of $B^{\prime}$ for each $B^{\prime} \in U$ and $\sigma(B)=s$. The set of (stable) symmetries of $B$ is denoted by $\operatorname{Sym} B$ (respectively, $\operatorname{Sym}_{\mathrm{st}} B$ ).

Alternatively, one can consider the bundle

$$
\operatorname{Sym}(\Sigma)=\left\{(B, s) \in \mathcal{C}_{m}(\Sigma) \times P G L(3, \mathbb{C}) \mid s \in \operatorname{Sym} B\right\} \rightarrow \mathcal{C}_{m}(\Sigma)
$$

and define the (nonabelian) sheaf $\operatorname{Sym}_{\mathrm{st}}(\Sigma)$ of germs of sections of $\operatorname{Sym}(\Sigma)$. Then $\operatorname{Sym}_{\mathrm{st}} B$ is the stalk of $\operatorname{Sym}_{\mathrm{st}}(\Sigma)$ over $B$. Note that a priori it is not obvious that $\operatorname{Sym}_{\mathrm{st}}(\Sigma)$ is locally constant or even that the groups $\operatorname{Sym}_{\mathrm{st}} B$ are semicontinuous in any reasonable sense. Below we show, see Corollary 2.5.4, that the sheaf $\operatorname{Sym}_{\text {st }}(\Sigma)$ is indeed locally constant in the case of curves of degree six with simple singularities only. Furthermore, we show that, in this case, the group of stable symmetries is the group of symmetries of a generic curve in a given equisingular stratum (see Corollary 2.4.4); thus, the study of $\operatorname{Sym}_{\mathrm{st}} B$ is equivalent to the study of a generic fiber $\operatorname{PGL}(3, \mathbb{C}) / \operatorname{Sym}_{\mathrm{st}} B$ of the projection $\mathcal{C}_{6}\left(\Sigma_{B}\right) \rightarrow \mathcal{M}_{6}\left(\Sigma_{B}\right)$.

It is worth mentioning that stable symmetries can also be used to describe the moduli space $\mathcal{M}_{6}\left(\Sigma_{B}\right)$ itself. Thus, from the results of [8], [9], and [10] it follows that, for all sets of singularities mentioned in Theorem 1.3.1(1), (3), (6), and (7) below, the moduli spaces are unirational. It is anticipated that a similar statement holds for (most of) the other sets of singularities listed in Theorem 1.3.1; we will discuss this in details in a subsequent paper.

1.3. Principal results. In order to state our principal result, we introduce a few terms. A $\mathbb{D}_{2 p}$-sextic is an irreducible plane sextic $B$ with simple singularities and such that the fundamental group $\pi_{1}\left(\mathbb{P}^{2} \backslash B\right)$ factors to the dihedral group $\mathbb{D}_{2 p}$. As

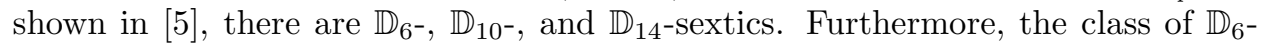
sextics coincides with the class of irreducible sextics with simple singularities that are of torus type, i.e., whose equation can be represented in the form $p^{3}+q^{2}=0$, where $p$ and $q$ are some homogeneous polynomials of degree 2 and 3, respectively.

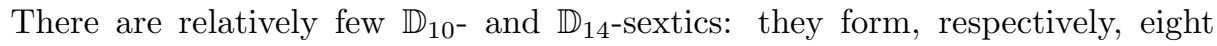
and two deformation families, see [5]. In order to describe the hierarchy of $\mathbb{D}_{6^{-}}$ sextics, we introduce the weight $w(B)$ as the total weight of the singularities of $B$, where the weight of a simple singular point $P$ is defined as follows: $w\left(\mathbf{A}_{3 i-1}\right)=i$, $w\left(\mathbf{E}_{6}\right)=2$, and $w(P)=0$ otherwise. One has $w(B) \leqslant 9$, if $B$ is of torus type, then $w(B) \geqslant 6$, and a sextic of weight $\geqslant 6$ is of torus type unless $w(B)=6$ and all singular points of $B$ of weight zero are nodes. In the latter exceptional case, most sets of singularities are realized by two deformation families, one of torus type and one not; for the complete classification, see A. Özgüner [14].

If $B$ is a plane sextic of torus type and $p^{3}+q^{2}$ is a torus structure of $B$, denote by $\Sigma_{B}^{\text {in }}$ the set of inner singularities of $B$. (Recall that a singular point $P$ of $B$ 
is called inner with respect to a given torus structure $p^{3}+q^{2}$ if $P$ belongs to the intersection of the conic $\{p=0\}$ and the cubic $\{q=0\}$.)

The principal result of the present paper is the following theorem, which gives a complete classification of stable symmetries of irreducible plane sextics with simple singularities.

1.3.1. Theorem. The following is the complete list of irreducible plane sextics with simple singularities and nontrivial group $\operatorname{Sym}_{\mathrm{st}} B$ of stable symmetries:

(1) all sextics of weight nine: $\operatorname{Sym}_{\mathrm{st}} B=\left(\mathbb{Z}_{3} \times \mathbb{Z}_{3}\right) \rtimes \mathbb{Z}_{2}$, the $\mathbb{Z}_{2}$ factor acting on the kernel $\mathbb{Z}_{3} \times \mathbb{Z}_{3}$ via the multiplication by $(-1)$;

(2) $\mathbb{D}_{6}$-sextics with $\Sigma_{B}^{\text {in }}=3 \mathbf{E}_{6}: \operatorname{Sym}_{\mathrm{st}} B$ is the symmetric group $\mathbb{S}_{3}$;

(3) all $\mathbb{D}_{14}$-sextics: $\mathrm{Sym}_{\mathrm{st}} B=\mathbb{Z}_{3}$.

For the rest of the list, one has $\operatorname{Sym}_{\mathrm{st}} B=\mathbb{Z}_{2}$ :

(4) $\mathbb{D}_{6}$-sextics with $\Sigma_{B}^{\text {in }}=2 \mathbf{E}_{6} \oplus \mathbf{A}_{5}$ or $2 \mathbf{E}_{6} \oplus 2 \mathbf{A}_{2}$;

(5) $\mathbb{D}_{6}$-sextics with $\Sigma_{B}^{\text {in }}=\mathbf{A}_{17}$ or $2 \mathbf{A}_{8}$;

(6) all sextics of weight eight;

(7) all $\mathbb{D}_{10}$-sextics;

(8) sextics with $\Sigma_{B}=2 \mathbf{E}_{8} \oplus \Sigma^{\prime}$, where $\Sigma^{\prime}=\mathbf{A}_{3}, \mathbf{A}_{2}$, or $k \mathbf{A}_{1}, k=0,1,2$.

Theorem 1.3.1 is proved in Section 3.8.

1.3.2. Remark. In items (2), (4), and (5), the curves have weight $\leqslant 7$; hence, each curve $B$ has a unique torus structure, see [5], and $\Sigma_{B}^{\text {in }}$ is well defined. Item (8) lists all sextics with two type $\mathbf{E}_{8}$ singular points, see Proposition 3.1.2.

There is a mysterious connection between stable involutions of irreducible plane sextics and maximal (in the sense of [5], see Definition 4.1.2 for details) trigonal curves in the cone $\Sigma_{2}$. Roughly, an involution $c \in \operatorname{Sym} B$ is stable if and only if the quotient $B / c$ is maximal. We postpone the precise statement till $\S 4$, see Theorems 4.2.1 and 4.2.2, as they require a number of preliminary definitions.

1.4. Contents of the paper. In $\S 2$, we reduce the problem of classification of stable symmetries to a combinatorial question. First, we apply the theory of $K 3$ surfaces and describe the symmetries of plane sextics with simple singularities in arithmetical terms, see Theorem 2.3.7. Next, in Theorem 2.4.1, we give an arithmetical characterization of stable symmetries. With a few exceptions with the maximal total Milnor number $\mu=19$, this theorem applies to reducible curves as well. Finally, in Theorem 2.5.3, we describe stable symmetries of irreducible sextics in terms of symmetries of their Dynkin graphs. With the few exceptions above, this theorem also applies to reducible curves, provided that the definition of configuration and its stable symmetry is modified to take into account the hyperplane section class.

In $\S 3$, we classify stable symmetries of Dynkin graphs of irreducible sextics and prove Theorem 1.3.1.

$\S 4$ deals with stable involutions and trigonal curves. First, we classify all stable maximal trigonal curves in the Hirzebruch surface $\Sigma_{2}$, see Theorem 4.1.3. Then, comparing this result and Theorem 1.3.1, we give a characterization of stable involutions of irreducible plane sextics in terms of the maximality of the quotient curve. There is strong evidence that a similar relation holds as well for reducible sextics with simple singularities, see Conjecture 4.2.3 and Remark 4.2.5. 


\section{The COMBINATORIAL REDUCTION}

The principal result of this section is Theorem 2.5.3, reducing the study of stable symmetries of plane sextics to the study of symmetries of their Dynkin diagrams.

2.1. Discriminant forms. An (integral) lattice is a finitely generated free abelian group $S$ supplied with a symmetric bilinear form $b: S \otimes S \rightarrow \mathbb{Z}$. We abbreviate $b(x, y)=x \cdot y$ and $b(x, x)=x^{2}$. A lattice $S$ is called even if $x^{2}=0 \bmod 2$ for all $x \in S$. As the transition matrix between two integral bases has determinant \pm 1 , the determinant $\operatorname{det} S \in \mathbb{Z}$ (i.e., the determinant of the Gram matrix of $b$ in any basis of $S$ ) is well defined. A lattice $S$ is called nondegenerate if $\operatorname{det} S \neq 0$; it is called unimodular if $\operatorname{det} S= \pm 1$.

Given a lattice $S$, the form $b$ extends to a form $(S \otimes \mathbb{Q}) \otimes(S \otimes \mathbb{Q}) \rightarrow \mathbb{Q}$. If $S$ is nondegenerate, the dual group $S^{*}=\operatorname{Hom}(S, \mathbb{Z})$ can be identified with the subgroup

$$
\{x \in S \otimes \mathbb{Q} \mid x \cdot y \in \mathbb{Z} \text { for all } x \in S\}
$$

In particular, $S \subset S^{*}$ is a finite index subgroup. The quotient $S^{*} / S$ is called the discriminant group of $S$ and is denoted by $\operatorname{discr} S$ or $\mathcal{S}$. The discriminant group inherits from $S \otimes \mathbb{Q}$ a symmetric bilinear form $b_{\mathcal{S}}: \mathcal{S} \otimes \mathcal{S} \rightarrow \mathbb{Q} / \mathbb{Z}$, called the discriminant form, and, if $S$ is even, its quadratic extension $q_{\mathcal{S}}$, i.e., a function $q_{\mathcal{S}}: \mathcal{S} \rightarrow \mathbb{Q} / 2 \mathbb{Z}$ such that $q_{\mathcal{S}}(x+y)=q_{\mathcal{S}}(x)+q_{\mathcal{S}}(y)+2 b_{\mathcal{S}}(x, y)$ for all $x, y \in \mathcal{S}$, where 2 is regarded as a homomorphism $\mathbb{Q} / \mathbb{Z} \rightarrow \mathbb{Q} / 2 \mathbb{Z}$. One has $|\mathcal{S}|=|\operatorname{det} S|$; in particular, $\mathcal{S}=0$ if and only if $S$ is unimodular.

Given a prime $p$, we use the notation $\operatorname{discr}_{p} S=\mathcal{S}_{p}$ for the $p$-primary part of $\mathcal{S}$. One has $\mathcal{S}_{p}=\mathcal{S} \otimes \mathbb{Z}_{p^{r}}, r \gg 1$, and $\mathcal{S}=\bigoplus_{p} \mathcal{S}_{p}$, the sum running over all primes.

From now on, all lattices considered are even and nondegenerate.

An extension of a lattice $S$ is another lattice $M$ containing $S$, so that the form on $S$ is the restriction of that on $M$. An isomorphism between two extensions $M_{1} \supset S$ and $M_{2} \supset S$ is an isometry $M_{1} \rightarrow M_{2}$ whose restriction to $S$ is the identity. Next three theorems are found in Nikulin [11].

2.1.1. Theorem. Given a lattice $S$, there is a canonical one-to-one correspondence between the set of isomorphism classes of finite index extensions $M \supset S$ and the set of isotropic subgroups $\mathcal{K} \subset \mathcal{S}$. Under this correspondence, one has $M=\left\{x \in S^{*} \mid x \bmod S \in \mathcal{K}\right\}$ and $\operatorname{discr} M=\mathcal{K}^{\perp} / \mathcal{K}$.

The isotropic subgroup $\mathcal{K} \subset \mathcal{S}$ as in Theorem 2.1.1 is called the kernel of the extension $M \supset S$. It can be defined as the image of $M / S$ under the homomorphism induced by the natural inclusion $M \hookrightarrow S^{*}$.

2.1.2. Theorem. Let $M \supset S$ be a finite index extension of a lattice $S$, and let $\mathcal{K} \subset \mathcal{S}$ be its kernel. Then, an auto-isometry $S \rightarrow S$ extends to $M$ if and only if the induced automorphism of $\mathcal{S}$ preserves $\mathcal{K}$.

2.1.3. Theorem. Let $S \subset M$ be a primitive sublattice of a unimodular lattice $M$. Then the kernel of the finite index extension $M \supset S \oplus S^{\perp}$ is the graph of an antiisometry $\operatorname{discr} S \rightarrow \operatorname{discr} S^{\perp}$.

We will use Theorems 2.1.2 and 2.1.3 in the following form.

2.1.4. Corollary. Let $S \subset M$ be a sublattice of a unimodular lattice $M$, and let $\mathcal{K} \subset \mathcal{S}$ be the kernel of the extension $\tilde{S} \supset S$, where $\tilde{S}$ is the primitive hull of $S$ 
in $M$. Consider an auto-isometry $c: S \rightarrow S$. Then, $c \oplus \operatorname{id}_{S^{\perp}}$ extends to $M$ if and only if $c$ preserves $\mathcal{K}$ and the auto-isometry of $\mathcal{K}^{\perp} / \mathcal{K}$ induced by $c$ is trivial.

Proof. Apply Theorem 2.1.2 twice: first, to $\tilde{S} \supset S$, then to $M \supset \tilde{S} \oplus S^{\perp}$.

2.2. Root systems. A root in an even lattice $S$ is an element $v \in S$ of square -2 . A root system is a negative definite lattice generated by its roots. Every root system admits a unique decomposition into an orthogonal sum of irreducible root systems, the latter being either $\mathbf{A}_{p}, p \geqslant 1$, or $\mathbf{D}_{q}, q \geqslant 4$, or $\mathbf{E}_{6}, \mathbf{E}_{7}, \mathbf{E}_{8}$. The discriminant forms are as follows:

$$
\begin{gathered}
\operatorname{discr} \mathbf{A}_{p}=\left[-\frac{p}{p+1}\right], \quad \operatorname{discr} \mathbf{D}_{2 k+1}=\left[-\frac{2 k+1}{4}\right], \\
\operatorname{discr} \mathbf{D}_{8 k \pm 2}=2\left[\mp \frac{1}{2}\right], \quad \operatorname{discr} \mathbf{D}_{8 k}=\mathcal{U}_{2}, \quad \operatorname{discr} \mathbf{D}_{8 k+4}=\mathcal{V}_{2}, \\
\operatorname{discr} \mathbf{E}_{6}=\left[\frac{2}{3}\right], \quad \operatorname{discr} \mathbf{E}_{7}=\left[\frac{1}{2}\right], \quad \operatorname{discr} \mathbf{E}_{8}=0 .
\end{gathered}
$$

Here, $\left[\frac{p}{q}\right]$ is the cyclic group $\mathbb{Z}_{q}$ generated by an element of square $\frac{p}{q} \in \mathbb{Q} / 2 \mathbb{Z}$, and $\mathcal{U}_{2}$ (respectively, $\mathcal{V}_{2}$ ) is the quadratic form on $\mathbb{Z}_{2} \oplus \mathbb{Z}_{2}$ generated by elements $x, y$ with $x \cdot y=\frac{1}{2} \in \mathbb{Q} / \mathbb{Z}$ and $x^{2}=y^{2}=0 \in \mathbb{Q} / 2 \mathbb{Z}$ (respectively, $x^{2}=y^{2}=1$ ).

Given a root system $S$, the group generated by reflections (defined by the roots of $S$ ) acts simply transitively on the set of Weyl chambers of $S$. The roots defining the walls of any Weyl chamber form a standard basis for $S$. The incidence graph $\Gamma$ of a standard basis is called the Dynkin diagram of $S$. Irreducible root systems correspond to connected Dynkin diagrams. With a certain abuse of the language, we will speak about the discriminant group $\operatorname{discr} \Gamma$ of a Dynkin diagram $\Gamma$, referring to the discriminant group of the root system $S_{\Gamma}$ spanned by $\Gamma$.

Denote by Sym $\Gamma$ the group of symmetries of a Dynkin diagram $\Gamma$. There is an obvious homomorphism

$$
\text { discr: } \operatorname{Sym} \Gamma \rightarrow O\left(S_{\Gamma}\right) \rightarrow \text { Aut discr } \Gamma \text {. }
$$

The following three statements are well known; they follow immediately from the classification of connected Dynkin diagrams, see, e.g., N. Bourbaki [2].

2.2.2. Lemma. Let $\Gamma$ be a connected Dynkin diagram. Then:

(1) $\operatorname{discr} \Gamma \neq 0$ unless $\Gamma$ is of type $\mathbf{E}_{8}$;

(2) the homomorphism discr: $\operatorname{Sym} \Gamma \rightarrow$ Aut discr $\Gamma$ is monic.

2.2.3. Lemma. Let $\Gamma$ be a connected Dynkin diagram. Then:

(1) if $\Gamma$ is of type $\mathbf{D}_{4}$, then $\operatorname{Sym} \Gamma=$ Aut discr $\Gamma=\mathbb{S}_{3}$;

(2) if $\Gamma$ is of type $\mathbf{A}_{1}, \mathbf{E}_{7}$, or $\mathbf{E}_{8}$, then $\operatorname{Sym} \Gamma=\operatorname{Aut} \operatorname{discr} \Gamma=1$;

(3) for all other types, $\operatorname{Sym} \Gamma=\mathbb{Z}_{2}$.

2.2.4. Lemma. If $\Gamma$ is a connected Dynkin diagram of type $\mathbf{A}_{p}, p \geqslant 2, \mathbf{D}_{2 k+1}$, or $\mathbf{E}_{6}$, then the only nontrivial symmetry of $\Gamma$ induces - id on discr $\Gamma$.

Further details on irreducible root systems are found in Bourbaki [2].

2.3. The covering $K 3$-surface. Let $B$ be a plane sextic with simple singularities. Denote by $X_{B} \rightarrow \mathbb{P}^{2}$ the double covering ramified at $B$, and let $\tilde{X}_{B}$ be the minimal resolution of $X_{B}$. It is a $K 3$-surface. Let $\tau: \tilde{X} \rightarrow \tilde{X}$ be the deck translation of the covering $\tilde{X}_{B} \rightarrow \mathbb{P}^{2}$, and let $\mathrm{Aut}_{\tau} \tilde{X}_{B}$ be the centralizer of $\tau$ in the group of automorphisms of $\tilde{X}_{B}$. Since any symmetry of $B$ lifts to two automorphisms of $\tilde{X}$, there is an exact sequence

$$
1 \rightarrow\{\mathrm{id} \tau\} \rightarrow \operatorname{Aut}_{\tau} \tilde{X}_{B} \rightarrow \operatorname{Sym} B \rightarrow 1 .
$$


2.3.2. Definition. The homological type of a sextic $B$ with simple singularities is the triple $\mathcal{H}_{B}=\left(L_{B}, h_{B}, \Gamma_{B}\right)$, where $L_{B}$ is the lattice $H_{2}\left(\tilde{X}_{B}\right), h_{B} \in L_{B}$ is the class of the pull-back of a generic line (so that $h^{2}=2$ ), and $\Gamma_{B}$ is the set of classes of the exceptional divisors over the singular points of $B$.

An automorphism of the homological type $\mathcal{H}_{B}=\left(L_{B}, h_{B}, \Gamma_{B}\right)$ is an isometry of $L_{B}$ preserving $h_{B}$ and $\Gamma_{B}$ (as a set). The group of automorphisms of $\mathcal{H}_{B}$ is denoted by Aut $\mathcal{H}_{B}$. We will also consider the subgroups Aut $\mathcal{H}_{B}$ and $\mathrm{Aut}_{ \pm} \mathcal{H}_{B}$ consisting of the automorphisms inducing, respectively, id and \pm id on the orthogonal complement $\left(\Gamma_{B} \cup h_{B}\right)^{\perp}$.

Denote by $\Sigma_{B} \subset L_{B}$ the sublattice spanned by $\Gamma_{B}$, and let $S_{B}=\Sigma_{B} \oplus\left\langle h_{B}\right\rangle$. The primitive hulls of $\Sigma_{B}$ and $S_{B}$ in $L_{B}$ are denoted by $\tilde{\Sigma}_{B}$ and $\tilde{S}_{B}$, respectively, and the kernel of the finite index extension $\tilde{S}_{B} \supset S_{B}$ is denoted by $\mathcal{K}_{B}$.

2.3.3. Lemma. Aut $\mathcal{H}_{B}$ is the subgroup of elements $s \in$ Aut $\mathcal{H}_{B}$ inducing a scalar on $S_{\bar{B}}^{\perp}$.

Proof. For any $s \in$ Aut $\mathcal{H}_{B}$, both the restriction $\left.s\right|_{S^{\perp}}$ and its inverse are defined over $\mathbb{Z}$; hence, if $\left.s\right|_{S^{\perp}}$ is a scalar, one must have $\left.s\right|_{S^{\perp}}= \pm \mathrm{id}$.

The following statement is contained in [4].

2.3.4. Proposition. If $B$ is irreducible, then $\mathcal{K}_{B}$ is free of 2-torsion. In particular, one has $\mathcal{K}_{B} \subset \operatorname{discr} \Sigma_{B}, \tilde{S}_{B}=\tilde{\Sigma}_{B} \oplus\left\langle h_{B}\right\rangle$, and discr $\tilde{S}_{B}=\operatorname{discr} \tilde{\Sigma}_{B} \oplus\left\langle\frac{1}{2} h_{B}\right\rangle$.

The lattice $\Sigma_{B}$ is a root system, and the elements of $\Gamma_{B}$ form a standard basis for $\Sigma_{B}$, see 2.2. In what follows, we identify the set $\Gamma_{B}$ with its incidence graph. The connected components of $\Gamma_{B}$ (irreducible components of $\Sigma_{B}$ ) are in a one-to-one correspondence with the singular points of $B$, each component being a connected Dynkin diagram (respectively, irreducible root system) of the same name as the type of the singular point.

2.3.5. Definition. The period of a sextic $B$ is the 1 -subspace $\omega_{B}=H^{2,0}\left(\tilde{X}_{B}\right) \subset$ $L_{B} \otimes \mathbb{C}$; it is formed by the classes of holomorphic 2-forms on $\tilde{X}_{B}$. The extended homological type of $B$ is the pair $\left(\mathcal{H}_{B}, \omega_{B}\right)$. An automorphism of the extended homological type is an automorphism of $\mathcal{H}_{B}$ preserving $\omega_{B}$. The group of automorphisms of $\left(\mathcal{H}_{B}, \omega_{B}\right)$ is denoted by $\operatorname{Aut}\left(\mathcal{H}_{B}, \omega_{B}\right)$.

Recall that the period $\omega_{B}$ is a point in the projectivization of the cone

$$
\Omega=\left\{x \in S_{B}^{\perp} \otimes \mathbb{C} \mid x^{2}=0, x \cdot \bar{x}>0\right\} .
$$

Conversely, any generic (complementary to a countable union of hyperplanes) point in the projectivization of $\Omega$ is the period of a certain sextic, which is in the same equisingular stratum $\mathcal{C}_{6}\left(\Sigma_{B}\right)$ as $B$.

There are obvious inclusions

$$
\text { Aut }_{+} \mathcal{H}_{B} \subset \operatorname{Aut}_{ \pm} \mathcal{H}_{B} \subset \operatorname{Aut}\left(\mathcal{H}_{B}, \omega_{B}\right) \subset \operatorname{Aut} \mathcal{H}_{B}
$$

By definition, $\omega_{B}$ is an eigenspace of any element $a \in \operatorname{Aut}\left(\mathcal{H}_{B}, \omega_{B}\right)$. Sending $a$ to the corresponding eigenvalue defines a homomorphism $\lambda: \operatorname{Aut}\left(\mathcal{H}_{B}, \omega_{B}\right) \rightarrow \mathbb{C}^{*}$.

Any automorphism $\tilde{c} \in \operatorname{Aut}_{\tau} \tilde{X}_{B}$ induces an automorphism $\tilde{c}_{*} \in \operatorname{Aut}\left(\mathcal{H}_{B}, \omega_{B}\right)$. In particular, $\tau$ itself induces an automorphism $\tau_{*} \in \operatorname{Aut}\left(\mathcal{H}_{B}, \omega_{B}\right)$, which can be 
described as follows. On each connected component of $\Gamma_{B}$ of type $\mathbf{A}_{p}, p \geqslant 2$, $\mathbf{D}_{2 k+1}$, or $\mathbf{E}_{6}, \tau_{*}$ is the only nontrivial symmetry, on any other component and on $\left\langle h_{B}\right\rangle$, it is the identity, and on $S_{B}^{\perp}$, minus identity. The map just described preserves $\mathcal{K}_{B}$ (as well as any subgroup of $\operatorname{discr} S_{B}$ ), and the induced automorphisms of all discriminants are - id; due to Theorem 2.1.2, the map extends to $L_{B}$.

2.3.7. Theorem. For any plane sextic $B \in \mathbb{P}^{2}$ with simple singularities, the map $\tilde{c} \mapsto \tilde{c}_{*}$ establishes an isomorphism $\operatorname{Aut}_{\tau} \tilde{X}_{B}=\operatorname{Aut}\left(\mathcal{H}_{B}, \omega_{B}\right)$. Hence, there is an exact sequence

$$
1 \rightarrow\left\{\mathrm{id}, \tau_{*}\right\} \rightarrow \operatorname{Aut}\left(\mathcal{H}_{B}, \omega_{B}\right) \rightarrow \operatorname{Sym} B \rightarrow 1
$$

obtained from (2.3.1) via the above isomorphism.

Proof. Let Pic $\tilde{X}_{B}=\omega_{B}^{\perp} \cap L_{B}$ be the Picard group of $\tilde{X}_{B}$. Recall that the Kähler cone $V_{B}^{+}$of $\tilde{X}_{B}$ can be defined as the set

$$
\left\{x \in \omega_{B}^{\perp} \cap\left(L_{B} \otimes \mathbb{R}\right) \mid x^{2}>0 \text { and } x \cdot[E]>0 \text { for any }(-2) \text {-curve } E \subset \tilde{X}_{B}\right\} \text {. }
$$

The projectivization $\mathbb{P}\left(V_{B}^{+}\right)$is an (open) fundamental polyhedron of the group of motions of the hyperbolic space $\mathbb{P}\left(\left\{x \in \omega_{B}^{\perp} \cap\left(L_{B} \otimes \mathbb{R}\right) \mid x^{2}>0\right\}\right)$ generated by the reflections defined by the roots of $\mathrm{Pic} \tilde{X}_{B}$. In the case under consideration, $V_{B}^{+}$is characterized (among the other fundamental polyhedra) by the following properties:

(1) $V_{B}^{+} \cdot v>0$ for any $v \in \Gamma_{B}$;

(2) the closure of $V_{B}^{+}$contains $h_{B}$.

Consider an element $\tilde{c}_{*} \in \operatorname{Aut}\left(\mathcal{H}_{B}, \omega_{B}\right)$ and regard it as an isometry of $H_{2}\left(\tilde{X}_{B}\right)$. By definition, $\tilde{c}_{*}$ preserves $h_{B}, \Gamma_{B}$, and $\omega_{B}$; hence, $\tilde{c}_{*}$ also preserves $V_{B}^{+}$. Now, a standard argument using the description of the fine period space of marked Kähler $K 3$-surfaces, see A. Beauville [1], shows that any isometry $\tilde{c}_{*}$ of $H_{2}\left(\tilde{X}_{B}\right)$ preserving $\omega_{B}$ and $V_{B}^{+}$is induced by a unique automorphism $\tilde{c}$ of $\tilde{X}_{B}$. Since $\tilde{c}_{*}\left(h_{B}\right)=h_{B}$ and $h_{B}$ (regarded as an element of Pic $\tilde{X}_{B}$ ) is the linear system defining the projection $\tilde{X} \rightarrow \mathbb{P}^{2}$, one has $\tilde{c} \in$ Aut $_{\tau} \tilde{X}_{B}$. The existence (uniqueness) of $\tilde{c}$ above assert that the map $\tilde{c} \mapsto \tilde{c}_{*}$ is onto (respectively, one-to-one).

2.3.8. Proposition. For any sextic B, the group Sym $B$ is finite.

Proof. Since $\tilde{X}_{B}$ is obviously algebraic, the kernel of the canonical representation Aut $\tilde{X}_{B} \rightarrow O\left(\operatorname{Pic} \tilde{X}_{B}\right)$ is a finite cyclic group, see Nikulin [12]. On the other hand, the image of Aut $\tilde{X}_{B}$ is a subgroup of $O\left(h_{B}^{\perp} \cap \operatorname{Pic} \tilde{X}_{B}\right)$. Since $h \frac{\perp}{B} \cap \operatorname{Pic} \tilde{X}_{B}$ is a negative definite lattice, its group of isometries is finite.

2.4. The symplectic lift. Recall that an automorphism of a $K 3$-surface $X$ is called symplectic (anti-symplectic) if it preserves (respectively, reverses) holomorphic 2-forms on $X$. Note that any automorphism multiplies all 2 -forms by a certain constant $\lambda \in \mathbb{C}^{*}$; the automorphism is (anti-)symplectic if and only if $\lambda= \pm 1$.

2.4.1. Theorem. Let $B \subset \mathbb{P}^{2}$ be a sextic with simple singularities, and assume that either $\mu(B)<19$ or $B$ is irreducible. Then, for any stable symmetry $c$ of $B$, one of the two lifts of $c$ to the covering $K 3$-surface $\tilde{X}_{B}$ is symplectic, and the other one is anti-symplectic. The induced automorphisms of $\mathcal{H}_{B}$ belong to Aut $\pm \mathcal{H}_{B}$. 
Proof. Since the two lifts differ by $\tau$, which is anti-symplectic and induces - id on $S_{B}^{\perp}$, it suffices to show that any lift induces \pm id on $S_{B}^{\perp}$. (Then it acts via $( \pm 1)$ on $\omega_{B}$ and, hence, is symplectic or anti-symplectic.)

Let $\tilde{c}_{*}$ be the automorphism of $S_{B}^{\perp}$ induced by the chosen lift. Then the period $\omega_{B} \subset S_{B}^{\perp} \otimes \mathbb{C}$ is an eigenspace of $\tilde{c}_{*}$. Assume that $\operatorname{rk} S_{B}^{\perp} \geqslant 3$. Since $c$ is stable, there is a neighborhood $U$ of $\omega_{B}$ in the projectivization of the cone $\Omega$, see (2.3.6), such that any generic $\omega \in U$ is also an eigenspace of $\tilde{c}_{*}$, obviously corresponding to the same eigenvalue as $\omega_{B}$. On the other hand, any open subset of $\Omega$ spans $S_{B}^{\perp} \otimes \mathbb{C}$. Thus, $\tilde{c}_{*}$ is a scalar and Lemma 2.3.3 applies.

Now, assume that $\mathrm{rk} S_{B}^{\perp}=2$. Any positive definite lattice of rank 2 admitting an orientation preserving automorphism other than \pm id is isomorphic to either $\mathbf{A}_{2}(-m)$ or $2 \mathbf{A}_{1}(-m)$, where $m$ is a positive integer and $S(m)$ means that the bilinear form on the lattice $S$ is multiplied by $m$. (For example, one can argue that these are the lattices in $\mathbb{C}^{1}=\mathbb{R}^{2}$ admitting a non-trivial complex multiplication.) On the other hand, since $B$ is irreducible, the discriminant discr $\tilde{S}_{B} \cong-\operatorname{discr} S_{B}^{\perp}$ (see Theorem 2.1.3) has a direct summand $\left\langle\frac{1}{2} h_{B}\right\rangle \cong\left[\frac{1}{2}\right]$, see Proposition 2.3.4. It is immediate that neither discr $\mathbf{A}_{2}(-m)$ nor discr $2 \mathbf{A}_{1}(-m), m>0$, has a direct summand $\left[-\frac{1}{2}\right]$.

2.4.2. Example. There do exist reducible plane sextics for which the conclusion of Theorem 2.4.1 fails. For example, take for $B$ the curve given, in some affine coordinates $(x, y)$, by the equation

$$
\left(y^{3}-y\right)\left(y^{3}-y+x^{3}\right)=0 .
$$

The set of singularities of $B$ is $\mathbf{D}_{4} \oplus 3 \mathbf{A}_{5}$; hence, $\mu(B)=19$ and $\operatorname{Sym}_{\mathrm{st}} B=\operatorname{Sym} B$. (Note also that $B$ is a sextic of torus type with four irreducible components; a simple calculation shows that $S_{B}^{\perp}=\mathbf{A}_{2}(-1)$.) An affine part of $X_{B}$ is given by

$$
z^{2}=\left(y^{3}-y\right)\left(y^{3}-y+x^{3}\right) .
$$

The lift $(x, y, z) \mapsto(\epsilon x, y, z)$ of the symmetry $(x, y) \mapsto(\epsilon x, y), \epsilon^{3}=1$, is an order 3 automorphism of $X_{B}$ with a dimension one component $\{x=0\}$ in the fixed point set; hence, it is neither symplectic nor anti-symplectic.

2.4.3. Proposition. For any automorphism $\tilde{c}_{*} \in \mathrm{Aut}_{ \pm} \mathcal{H}_{B}$, its image in Sym $B$, see Theorem 2.3.7, is stable.

Proof. For any sextic $B^{\prime}$ close to $B$ in its equisingular stratum $\mathcal{C}_{6}\left(\Sigma_{B}\right)$, one can identify $\mathcal{H}_{B^{\prime}}$ and $\mathcal{H}_{B}$ and, under this identification, $\omega_{B^{\prime}}$ is a 1 -space close to $\omega_{B}$ in $\mathbb{P}\left(S_{B}^{\perp} \otimes \mathbb{C}\right)$. Hence, $\tilde{c}_{*}$ is also an automorphism of $\left(\mathcal{H}_{B^{\prime}}, \omega_{B^{\prime}}\right)$, and $c$ is stable.

2.4.4. Corollary. If $B$ is generic in its equisingular stratum (i.e., $B$ belongs to the complement of a certain countable union of codimension 1 subsets of $\mathcal{C}_{m}\left(\Sigma_{B}\right)$ ), then $\operatorname{Sym}_{\mathrm{st}} B=\operatorname{Sym} B$.

Proof. If $\mu(B)=19$, the statement is obvious, as the moduli space $\mathcal{M}_{6}\left(\Sigma_{B}\right)$ is discrete. Otherwise, in view of Theorem 2.3.7 and Proposition 2.4.3, it suffices to show that $\operatorname{Aut}\left(\mathcal{H}_{B}, \omega\right)=\operatorname{Aut}_{ \pm} \mathcal{H}_{B}$ for a generic element $\omega \in \mathbb{P}(\Omega)$, see (2.3.6). The latter statement follows from the the fact that the group Aut $\mathcal{H}_{B}$ is countable and from Lemma 2.3.3, which implies that, for any $s \in$ Aut $\mathcal{H}_{B} \backslash \operatorname{Aut}_{ \pm} \mathcal{H}_{B}$, the eigenspaces of the restriction $\left.s\right|_{S^{\perp}}$ are proper subspaces of $S_{B}^{\perp}$. 
2.5. Reduction to Dynkin diagrams. From now on, we consider irreducible sextics only and reserve the notation $\tilde{c}$ for the symplectic lift of a stable symmetry $c$ to the covering $K 3$-surface $\tilde{X}_{B}$; it is well defined due to Theorem 2.4.1. We denote by $\tilde{c}_{*}$ the induced isometry of $L_{B}=H_{2}\left(\tilde{X}_{B}\right)$, and by $\tilde{c}_{\Gamma}: \Gamma_{B} \rightarrow \Gamma_{B}$, the induced symmetry of the Dynkin diagram.

2.5.1. Definition. The configuration of an irreducible plane sextic $B$ with simple singularities is the pair $\left(\Gamma_{B}, \mathcal{K}_{B}\right)$, where $\mathcal{K}_{B} \subset \operatorname{discr} \Gamma_{B}$ is the kernel of the extension $\tilde{\Sigma} \supset \Sigma$, see 2.3. A symmetry of $\left(\Gamma_{B}, \mathcal{K}_{B}\right)$ is a symmetry $s \in \operatorname{Sym} \Gamma_{B}$ such that discr $s$ preserves $\mathcal{K}_{B}$. A symmetry $s$ is called stable if discr $s$ acts identically on $\mathcal{K}_{B}^{\perp} / \mathcal{K}_{B}$.

The group of symmetries (stable symmetries) of the configuration $\left(\Gamma_{B}, \mathcal{K}_{B}\right)$ is denoted by $\operatorname{Sym}\left(\Gamma_{B}, \mathcal{K}_{B}\right)$ (respectively, $\operatorname{Sym}_{\mathrm{st}}\left(\Gamma_{B}, \mathcal{K}_{B}\right)$ ).

2.5.2. Remark. In [4], the configuration of a sextic $B$ is defined as the finite index extension $\tilde{S}_{B} \supset S_{B}=\Sigma_{B} \oplus\left\langle h_{B}\right\rangle$. In view of Proposition 2.3.4 and Theorem 2.1.1, in the case of irreducible sextics the two definitions are equivalent.

2.5.3. Theorem. For an irreducible plane sextic $B$ with simple singularities, the map $c \mapsto \tilde{c}_{\Gamma}$ establishes an isomorphism $\operatorname{Sym}_{\mathrm{st}} B \rightarrow \operatorname{Sym}_{\mathrm{st}}\left(\Gamma_{B}, \mathcal{K}_{B}\right)$.

Proof. In view of Proposition 2.4.3, the exact sequence given by Theorem 2.3.7 restricts to

$$
1 \rightarrow\left\{\mathrm{id}, \tau_{*}\right\} \rightarrow \operatorname{Aut}_{ \pm} \mathcal{H}_{B} \rightarrow \operatorname{Sym}_{\mathrm{st}} B .
$$

Theorem 2.4.1 provides a splitting $c \mapsto \tilde{c}_{*}$ and, hence, an isomorphism

$$
\operatorname{Sym}_{\mathrm{st}} B \stackrel{\cong}{\longrightarrow} \operatorname{Aut}_{+} \mathcal{H}_{B} \text {. }
$$

Any element $\tilde{c}_{*} \in$ Aut $_{+} \mathcal{H}_{B}$ is uniquely determined by its restriction to $\Gamma_{B}$, and a symmetry $\tilde{c}_{\Gamma} \in \operatorname{Sym} \Gamma_{B}$ extends to an element of Aut $\mathcal{H}_{B}$ if and only if it is a stable symmetry of $\left(\Gamma_{B}, \mathcal{K}_{B}\right)$, see Corollary 2.1.4.

2.5.4. Corollary. Up to isomorphism, the group $\operatorname{Sym}_{\mathrm{st}} B$ depends only on the configuration of $B$. Furthermore, any path $B_{t}, t \in[0,1]$, in the equisingular stratum $\mathcal{C}_{6}\left(\Sigma_{B}\right)$ induces an isomorphism $\operatorname{Sym}_{\mathrm{st}} B_{0} \rightarrow \operatorname{Sym}_{\mathrm{st}} B_{1}$.

\section{Proof of Theorem 1.3.1}

Throughout this section, $B$ is an irreducible plane sextic with simple singularities. We use the notation introduced in 2.3 , abbreviating $\tilde{X}_{B}=\tilde{X}, \Gamma_{B}=\Gamma$, etc.

3.1. Sextics with type $\mathbf{E}_{8}$ singular points. Here, we treat the exceptional, in a certain sense, case of curves that admit a stable symmetry but are not $\mathbb{D}_{2 p}$-sextics.

3.1.1. Proposition. Let $s \in \operatorname{Sym}_{\mathrm{st}}\left(\Gamma_{B}, \mathcal{K}_{B}\right)$ and $s \neq \mathrm{id}$. Then either

(1) $B$ has two type $\mathbf{E}_{8}$ singular points, and $s$ is the transposition of the two type $\mathbf{E}_{8}$ components of $\Gamma_{B}$, or

(2) $B$ is a $\mathbb{D}_{2 p}$-sextic, $p=3,5,7$, and discr $s \neq \mathrm{id}$,

the two cases being mutually exclusive.

Proof. Assume that discr $s \neq$ id. Then, in order to make the action on $\mathcal{K}_{B}^{\perp} / \mathcal{K}_{B}$ trivial, one must have $\mathcal{K}_{B} \neq 0$. According to [5], $B$ is a $\mathbb{D}_{2 p}$-sextic, $p=3,5$, 7 ; in particular, $B$ has no type $\mathbf{E}_{8}$ singular points. 
Now, assume that $\operatorname{discr} s=\mathrm{id}$. Then, in view of Lemma 2.2.2, $s$ can only permute two or more type $\mathbf{E}_{8}$ components of $\Gamma_{B}$; in particular, $B$ has at least two type $\mathbf{E}_{8}$ singular points. On the other hand, since $\mu(B) \leqslant 19$, the number of type $\mathbf{E}_{8}$ points is at most two.

Sextics with at least two type $\mathbf{E}_{8}$ singular points are easily classified using [4]; we merely state the final result.

3.1.2. Proposition. A sextic with two type $\mathbf{E}_{8}$ singular points can have one of the five sets of singularities listed in Theorem 1.3.1(8). Each set of singularities is realized by a single equisingular deformation family.

3.2. $\mathbb{D}_{2 p}$-sextics. Let $B$ be a $\mathbb{D}_{2 p}$-sextic, $p=3,5,7$. Then, according to [5], the group $\mathcal{K}_{B} \neq 0$ is an $\mathbb{F}_{p^{-v}}$ vector space. A singular point $P_{i}$ of $B$ and the corresponding connected component $\Gamma_{i}$ of $\Gamma_{B}$ is called essential (ordinary) if the projection of $\mathcal{K}_{B}$ to discr $\Gamma_{i}$ is non-zero (respectively, zero). Let $\bar{\Gamma}_{B}$ be the union of all essential components of $\Gamma_{B}$; it is obviously preserved by stable symmetries. Denote by

$$
\pi_{0}: \operatorname{Sym} \Gamma_{B} \rightarrow \mathbb{S}\left(\pi_{0}\left(\Gamma_{B}\right)\right) \quad \text { and } \quad \bar{\pi}_{0}: \operatorname{Sym}_{\mathrm{st}}\left(\Gamma_{B}, \mathcal{K}_{B}\right) \rightarrow \mathbb{S}\left(\pi_{0}\left(\bar{\Gamma}_{B}\right)\right)
$$

the corresponding representations in the symmetric groups. Consider also the representation

$$
\kappa: \operatorname{Sym}_{\mathrm{st}}\left(\Gamma_{B}, \mathcal{K}_{B}\right) \rightarrow G L\left(\mathcal{K}_{B}\right)
$$

sending a symmetry $s \in \operatorname{Sym}_{\mathrm{st}}\left(\Gamma_{B}, \mathcal{K}_{B}\right)$ to the restriction of discr $s$ to $\mathcal{K}_{B}$. Note that $\bar{\pi}_{0}(s)$ and $\kappa(s)$ are well defined on $\operatorname{Sym}\left(\Gamma_{B}, \mathcal{K}_{B}\right)$ and, hence, on $\operatorname{Aut}_{\tau} \tilde{X}_{B}$. Furthermore, there is a homomorphism

$$
\bar{\kappa}: \operatorname{Sym} B \rightarrow P G L\left(\mathcal{K}_{B}\right)=G L\left(\mathcal{K}_{B}\right) / \pm \text { id }
$$

defined due to the fact that $\tau_{*}$ induces - id on the discriminant.

3.2.4. Proposition. Let $B$ be a $\mathbb{D}_{2 p}$-sextic, $p=3,5,7$, and let $s \in \operatorname{Sym}_{\mathrm{st}}\left(\Gamma_{B}, \mathcal{K}_{B}\right)$ be a stable symmetry of $\Gamma_{B}$. Then:

(1) $s$ acts trivially on $\Gamma_{B} \backslash \bar{\Gamma}_{B}$;

(2) discr $s$ acts trivially on $\operatorname{discr}_{q} \Gamma_{B}$ for any prime $q \neq p$;

(3) $s$ acts trivially on each component of $\Gamma_{B}$ other than $\mathbf{A}_{p^{r}-1}, \mathbf{A}_{2 p^{r}-1}, r \geqslant 1$, or $\mathbf{E}_{6}$ (in the case $p=3$ );

(4) $\pi_{0}(s)$ preserves each component of $\Gamma_{B}$ other than $\mathbf{A}_{p^{r}-1}, r \geqslant 1$, or $\mathbf{E}_{6}$ (in the case $p=3$ ).

Proof. The first statement follows from the fact that the discriminant of the union of the ordinary components of $\Gamma_{B}$ survives as a direct summand in $\mathcal{K}_{B}^{\perp} / \mathcal{K}_{B}$, the fact that a $\mathbb{D}_{2 p}$-sextic has no singular points of type $\mathbf{E}_{8}$, see [5], and Lemma 2.2.2.

Similarly, since $\mathcal{K}_{B} \subset \operatorname{discr}_{p} \Gamma_{B}$, any other primary component $\operatorname{discr}_{q} \Gamma_{B}, q \neq p$, survives to $\mathcal{K}_{B}^{\perp} / \mathcal{K}_{B}$; this observation implies (2), and the last two statements follow from (2) and (2.2.1).

3.2.5. Lemma. Let $G$ be a finite abelian group and $s: G \rightarrow G$ an automorphism of order prime to $|G|$. Assume that $G$ has an invariant subgroup $H$ such that the induced actions on $H$ and $G / H$ are both trivial. Then $s=$ id.

Proof. For each element $g \in G$ one has $s g-g \in H$. Then, for some $r$ prime to $|G|$, one has $g=s^{r} g=g+r(s g-g)$; hence, $r(s g-g)=0$ and $s g-g=0$. 
3.2.6. Corollary. Let $B$ be a $\mathbb{D}_{2 p}$-sextic, $p=3,5,7$. Then the kernel of the homomorphism $\kappa: \operatorname{Sym}_{\mathrm{st}}\left(\Gamma_{B}, \mathcal{K}_{B}\right) \rightarrow G L\left(\mathcal{K}_{B}\right)$, see (3.2.2), is a p-group.

Proof. Let $s \in \operatorname{Sym}_{\mathrm{st}}\left(\Gamma_{B}, \mathcal{K}_{B}\right)$ be an element of order prime to $p$, and assume that $\left.(\operatorname{discr} s)\right|_{\mathcal{K}}=$ id. Consider the filtration $\operatorname{discr}_{p} \Sigma_{B} \supset \mathcal{K}_{B}^{\perp} \supset \mathcal{K}_{B}$. (Here, ${ }^{\perp}$ stands for the orthogonal complement in $\operatorname{discr}_{p} \Sigma_{B}$.) The action of discr $s$ on $\mathcal{K}_{B}$ is trivial by the assumption, the action on $\mathcal{K}_{B}^{\perp} / \mathcal{K}_{B}$ is trivial since $s \in \operatorname{Sym}_{\mathrm{st}}\left(\Gamma_{B}, \mathcal{K}_{B}\right)$, and the action on $\operatorname{discr}_{p} \Sigma_{B} / \mathcal{K}_{B}^{\perp}$ is trivial due to the isomorphism $\operatorname{discr}_{p} \Sigma_{B} / \mathcal{K}_{B}^{\perp}=$ $\operatorname{Hom}\left(\mathcal{K}_{B}, \mathbb{Q} / \mathbb{Z}\right)$ given by the discriminant bilinear form. Applying Lemma 3.2.5 twice, we conclude that the action of discr $s$ on $\operatorname{discr}_{p} \Sigma_{B}$ is trivial. Hence, $s=$ id due to Propositions 3.2.4(2) and 3.1.1.

3.2.7. Corollary. Let $B$ be a $\mathbb{D}_{2 p}$-sextic, $p=3,5$, 7, with $\ell\left(\mathcal{K}_{B}\right)=1$. Then the kernel of the homomorphism $\kappa: \operatorname{Sym}_{\mathrm{st}}\left(\Gamma_{B}, \mathcal{K}_{B}\right) \rightarrow \mathbb{F}_{p}^{*}=G L\left(\mathcal{K}_{B}\right)$ is a p-group.

3.2.8. Corollary. Let $B$ be as in Corollary 3.2.7, and let $s \in \operatorname{Sym}_{\mathrm{st}}\left(\Gamma_{B}, \mathcal{K}_{B}\right)$ be an element of order prime to $p$. If the fixed point set of $s$ contains an essential component of $\Gamma_{B}$, then $s=$ id.

Proof. Under the assumption, $\kappa(s)=1 \in \mathbb{F}_{p}^{*}$, and Corollary 3.2.6 applies.

3.2.9. Corollary. If $B$ is a sextic as in Corollary 3.2.7, then $\left|\operatorname{Ker} \bar{\pi}_{0}\right| \leqslant 2$.

Proof. In view of Proposition 3.2.4(1) and Corollary 3.2.8, any nontrivial element $s \in \operatorname{Ker} \bar{\pi}_{0}$ has the following properties:

(1) the restriction of $s$ to each ordinary component of $\Gamma_{B}$ is id, and

(2) the restriction of $s$ to each essential component of $\Gamma_{B}$ is nontrivial.

Since an essential component of $\Gamma_{B}$ cannot be of type $\mathbf{D}_{4}$ (e.g., due to (2.2.1) and Proposition 2.3.4), it has at most one nontrivial symmetry, see Lemma 2.2.3; hence, the two properties above determine $s$ uniquely.

3.2.10. Proposition. Let $B$ be as in Corollary 3.2.7, and let $s \in \operatorname{Sym}_{\mathrm{st}}\left(\Gamma_{B}, \mathcal{K}_{B}\right)$, $s \neq \mathrm{id}$, be an element of order 2 . Then $\bar{\pi}_{0}(s)$ has at most two orbits.

Proof. Let $V_{p} \subset \operatorname{discr} \Gamma_{B}$ be the $\mathbb{F}_{p}$-vector space of order $p$ elements, and let $s_{*}$ be the action of $\operatorname{discr} s$ on $V_{p}$. Denote by $V_{p}^{-}$the $(-1)$ eigenspace of $s_{*}$. Then, each orbit of $\bar{\pi}_{0}(s)$ contributes one to $\operatorname{dim} V_{p}^{-}$: each two element orbit contributes a regular $\mathbb{F}_{p}$-representation of $\mathbb{Z}_{2}$, and each one element orbit contributes a one dimensional representation - id due to Corollary 3.2.8 and Lemma 2.2.4. On the other hand, since $\operatorname{dim} \mathcal{K}_{B}=1$, the stability condition requires $\operatorname{dim} V_{p}^{-} \leqslant 2$.

3.2.11. Corollary. Let $B$ be as in Corollary 3.2.7, and let $\left|\pi_{0}\left(\bar{\Gamma}_{B}\right)\right| \geqslant 3$. Then Ker $\bar{\pi}_{0}=1$.

3.3. $\mathbb{D}_{10}$-sextics. All $\mathbb{D}_{10}$-sextics are classified in [5]: they form eight equisingular deformation families, their sets of essential singularities are $4 \mathbf{A}_{4}, \mathbf{A}_{9} \oplus 2 \mathbf{A}_{4}$, or $2 \mathbf{A}_{9}$, and for any such sextic $B$ one has $\mathcal{K}_{B}=\mathbb{Z}_{5}$.

Any symmetry of $\Gamma_{B}$ of order divisible by 5 would have to permute cyclically at least five isomorphic components of $\Gamma_{B}$. Hence, such a symmetry does not exist, and Corollary 3.2.7 implies that $\operatorname{Sym}_{\mathrm{st}}\left(\Gamma_{B}, \mathcal{K}_{B}\right) \subset \mathbb{F}_{5}^{*} \cong \mathbb{Z}_{4}$. Symmetries of order 2 are constructed in [8], and to show that $\operatorname{Sym}_{\mathrm{st}}\left(\Gamma_{B}, \mathcal{K}_{B}\right) \cong \mathbb{Z}_{2}$, it remains to rule out symmetries of order 4 .

Let $s \in \operatorname{Sym}_{\mathrm{st}}\left(\Gamma_{B}, \mathcal{K}_{B}\right)$ be an element of order 4. Applying Corollary 3.2.8 to $s^{2}$ and using Lemma 2.2.3, one concludes that $\bar{\pi}_{0}(s)$ has no fixed points. Then, due to 
Proposition 3.2.4(4), the essential singularities of $B$ are $4 \mathbf{A}_{4}$, and Proposition 3.2.10 applied to $s^{2}$ implies that $\bar{\pi}_{0}(s)$ is a cycle of length 4 . Thus, discr $s$ is a regular $\mathbb{F}_{5}$-representation of $\mathbb{Z}_{4}$; it has four distinct eigenspaces (one for each eigenvalue $\left.\lambda \in \mathbb{F}_{5}^{*}\right)$ and thus cannot be stable.

3.4. $\mathbb{D}_{14}$-sextics. According to $[5], \mathbb{D}_{14}$-sextics form two equisingular deformation families; the set of essential singularities of any such sextic $B$ is $3 \mathbf{A}_{6}$, and one has $\mathcal{K}_{B}=\mathbb{Z}_{7}$.

As in 3.3, the graph $\Gamma_{B}$ has no symmetries of order 7 and Corollary 3.2.7 implies that $\operatorname{Sym}_{\mathrm{st}}\left(\Gamma_{B}, \mathcal{K}_{B}\right) \subset \mathbb{F}_{7}^{*} \cong \mathbb{Z}_{6}$. Symmetries of order 3 are constructed in [10]. Assume that $s \in \operatorname{Sym}_{\mathrm{st}}\left(\Gamma_{B}, \mathcal{K}_{B}\right)$ is a nontrivial element of order 2. Then, due to Proposition 3.2.10 and Corollary 3.2.8, discr $s$ is the direct sum of a dimension one representation - id and a regular $\mathbb{F}_{7}$-representation of $\mathbb{Z}_{2}$; it is easy to see that this action has no isotropic invariant subspaces.

3.5. $\mathbb{D}_{6}$-sextics: symmetries of order 3 . The sets of singularities of $\mathbb{D}_{6}$-sextics (i.e., irreducible sextics of torus type) are classified in M. Oka, D. T. Pho [13]. If $w(B) \leqslant 7$, the set of essential singularities of $B$ is of the form

$$
\bigoplus_{i} k_{i} \mathbf{A}_{3 i-1} \oplus l \mathbf{E}_{6}, \quad \sum_{i} i k_{i}+2 l=6,
$$

and one has $\mathcal{K}_{B}=\mathbb{Z}_{3}$. (In this case, essential are the inner singular points with respect to the only torus structure of $B$.) If $w(B)=8$, then the set of essential singularities is

$$
\mathbf{E}_{6} \oplus \mathbf{A}_{5} \oplus 4 \mathbf{A}_{2}, \quad \mathbf{E}_{6} \oplus 6 \mathbf{A}_{2}, \quad 2 \mathbf{A}_{5} \oplus 4 \mathbf{A}_{2}, \quad \mathbf{A}_{5} \oplus 6 \mathbf{A}_{2}, \quad \text { or } \quad 8 \mathbf{A}_{2},
$$

and one has $\mathcal{K}_{B}=\mathbb{Z}_{3} \oplus \mathbb{Z}_{3}$. Finally, there is one deformation family of sextics of weight 9: their set of singularities is $9 \mathbf{A}_{2}$, all nine cusps being essential, and one has $\mathcal{K}_{B}=\mathbb{Z}_{3} \oplus \mathbb{Z}_{3} \oplus \mathbb{Z}_{3}$. Stable symmetries of sextics of weight 9 are described in [9]; for this reason, we ignore them here.

Let $B$ be a plane sextic with simple singularities (not necessarily a $\mathbb{D}_{6}$-sextic or even irreducible), and let $\tilde{c} \in \mathrm{Aut}_{\tau} \tilde{X}_{B}$ be a symplectic automorphism of order 3 . Denote by $\tilde{X}^{\prime}$ the minimal resolution of singularities of the quotient $\tilde{X}_{B} / \tilde{c}$; it is also a $K 3$-surface (since $\tilde{c}$ is symplectic). Let $\Gamma^{\prime}$ be the union of the components of $\Gamma_{B}$ fixed by $\pi_{0}\left(\tilde{c}_{\Gamma}\right)$.

3.5.3. Lemma. In the notation above, $\Gamma^{\prime}$ has the form

$$
\bigcup_{i} k_{i} \mathbf{A}_{i} \cup l \mathbf{D}_{4} \text {, where } f=\sum_{i} k_{i}(i+1)+2 l \leqslant 6 \text {. }
$$

The image in $\tilde{X}^{\prime}$ of the divisor represented by each type $\mathbf{A}_{i}$ (respectively, $\mathbf{D}_{4}$ ) component of $\Gamma^{\prime}$ is a union of (-2)-curves in $\tilde{X}^{\prime}$ whose incidence graph is $\mathbf{A}_{3 i+2}$ (respectively, $\left.\mathbf{E}_{6}\right)$. In addition, $\tilde{c}$ has $(6-f)$ isolated fixed points not in the $(-2)$ curves represented by the vertices of $\Gamma_{B}$.

Proof. Let $E_{j} \subset \tilde{X}_{B}$ be the divisor represented by a component $\Gamma_{j}$ fixed by $\tilde{c}_{\Gamma}$ pointwise. $E_{j}$ is a union of $(-2)$-curves, and each point of intersection of two distinct components of $E_{j}$ is a fixed point of $\tilde{c}$. On the other hand, any (-2)-curve preserved by $\tilde{c}$ contains exactly two fixed points of $\tilde{c}$. (Since $\tilde{c}$ is symplectic, its fixed points are isolated.) Hence, $\Gamma_{j}$ cannot be of type $\mathbf{D}$ or $\mathbf{E}$ (as otherwise $E_{j}$ 
would have a component with three fixed points, hence fixed pointwise), and if it is of type $\mathbf{A}_{i}$, then $E_{j}$ contains $(i+1)$ fixed points of $\tilde{c}$.

Now, let $\Gamma_{j} \subset \Gamma^{\prime}$ be a component not fixed by $\tilde{c}_{\Gamma}$ pointwise. According to Lemma 2.2.3, it must be of type $\mathbf{D}_{4}$, and the divisor $E_{j}$ represented by $\Gamma_{j}$ has a single component (corresponding to the central vertex of $\Gamma_{j}$ ) fixed by $\tilde{c}$; this component contains two fixed points of $\tilde{c}$.

As is well known (see, e.g., Nikulin [12]), any symplectic automorphism of order 3 has six fixed points. Combining this observation with the count above, one obtains the estimate on the number and types of the components of $\Gamma^{\prime}$. The calculation of the image in $\tilde{X}^{\prime}$ is straightforward: each fixed point of $\tilde{c}$ gives rise to a cusp in $\tilde{X}_{B} / \tilde{c}$, i.e., to two extra $(-2)$-curves in $\tilde{X}^{\prime}$.

3.5.4. Corollary. Let $B$ be a $\mathbb{D}_{6}$-sextic with $w(B) \leqslant 8$, and let $\tilde{c} \in \operatorname{Aut}_{\tau} \tilde{X}_{B}$ be a symplectic automorphism of order 3 . Then the set of orbits of $\bar{\pi}_{0}\left(\tilde{c}_{\Gamma}\right)$ is one of the following: $\left(3 \mathbf{E}_{6}\right),\left(3 \mathbf{A}_{5}\right)$, or $\left(3 \mathbf{A}_{2}\right) \cup\left(3 \mathbf{A}_{2}\right)$.

Proof. If $w(B) \leqslant 7$, the statement follows immediately from Lemma 3.5.3 and (3.5.1). Assume that $w(B)=8$. Due to Lemma 3.5.3 and (3.5.2), the orbits of $\bar{\pi}_{0}\left(\tilde{c}_{\Gamma}\right)$ are either $\left(\mathbf{A}_{2}\right) \cup\left(\mathbf{A}_{2}\right) \cup\left(3 \mathbf{A}_{2}\right) \cup\left(3 \mathbf{A}_{2}\right)$ or $\left(\mathbf{A}_{5}\right) \cup\left(3 \mathbf{A}_{2}\right) \cup\left(3 \mathbf{A}_{2}\right)$. Hence, the projection of the exceptional divisor to $\tilde{X}^{\prime}$ is a divisor $E \subset \tilde{X}^{\prime}$ with the incidence graph $2 \mathbf{A}_{8} \cup \mathbf{A}_{2} \cup \mathbf{A}_{2}$ or $\mathbf{A}_{17} \cup \mathbf{A}_{2} \cup \mathbf{A}_{2}$. It spans a negative definite lattice of rank $\geqslant 20$, which does not fit into $H_{2}\left(\tilde{X}^{\prime}\right)$.

3.5.5. Corollary. $A \mathbb{D}_{6}$-sextic of weight $\leqslant 8$ has a stable symmetry of order 3 if and only if its set of essential singularities is $3 \mathbf{E}_{6}$.

Proof. A cyclic permutation of three type $\mathbf{A}_{5}$ components cannot be stable due to Proposition 3.2.4(4). For two cycles of length 3 on six cusps, the induced action on $\operatorname{discr} \Gamma_{B}$ is a direct sum of two copies of a regular $\mathbb{F}_{3}$-representation of $\mathbb{Z}_{3}$; it cannot be stable since $\operatorname{dim} \mathcal{K}_{B}=1$. The existence of a stable symmetry of order 3 on the set of essential singularities $3 \mathbf{E}_{6}$ is obvious.

3.6. $\mathbb{D}_{6}$-sextics of weight $\leqslant 7$. Propositions 3.6 .1 below describes the image of the representation $\kappa: \operatorname{Sym}_{\mathrm{st}}\left(\Gamma_{B}, \mathcal{K}_{B}\right) \rightarrow \mathbb{F}_{3}^{*}=\mathbb{Z}_{2}$, see Corollary 3.2.7.

3.6.1. Proposition. Let $B$ be a $\mathbb{D}_{6}$-sextic, $w(B) \leqslant 7$, and let $s \in \operatorname{Sym}_{\mathrm{st}}\left(\Gamma_{B}, \mathcal{K}_{B}\right)$ be a nontrivial element of order 2 . Then the orbits of $\bar{\pi}_{0}(s)$ are as follows:

$$
\left(2 \mathbf{E}_{6}\right) \cup\left(\mathbf{E}_{6}\right), \quad\left(2 \mathbf{E}_{6}\right) \cup\left(\mathbf{A}_{5}\right), \quad\left(2 \mathbf{E}_{6}\right) \cup\left(2 \mathbf{A}_{2}\right), \quad\left(\mathbf{A}_{17}\right), \quad\left(2 \mathbf{A}_{8}\right) .
$$

Conversely, any set of orbits as above is realized by a stable symmetry of order 2 .

Proof. In view of Proposition 3.2.4(3), (4), each orbit is one of the following: $2 \mathbf{E}_{6}$, $\mathbf{E}_{6}, \mathbf{A}_{17}, 2 \mathbf{A}_{8}, \mathbf{A}_{8}, \mathbf{A}_{5}, 2 \mathbf{A}_{2}$, or $\mathbf{A}_{2}$. Due to Proposition 3.2.10, there are at most two orbits. Combining these observations with (3.5.1), one obtains the five sets of orbits listed in the statement and $\left(\mathbf{A}_{8}\right) \cup\left(\mathbf{A}_{8}\right)$. In the latter case, disregarding the ordinary components, one has discr $s=-$ id, see Corollary 3.2.8 and Lemma 2.2.4, and since $\mathcal{K}_{B}^{\perp} / \mathcal{K}_{B} \cong \mathbb{Z}_{9}$, the symmetry is not stable.

The converse statement is straightforward: all five involutions are easily constructed using the description of $\mathcal{K}_{B}$ given in [5]. 
3.6.2. Corollary. Let $B$ be a $\mathbb{D}_{6}$-sextic of weight $w(B) \leqslant 7$. The representation $\kappa: \operatorname{Sym}_{\mathrm{st}} B \rightarrow \mathbb{F}_{3}^{*}=\mathbb{Z}_{2}$ is an epimorphism if and only if the set $\Sigma_{B}^{\text {in }}$ of essential singularities of $B$ is as in Theorem 1.3.1(2), (4), or (5). The kernel Ker $\kappa$ is trivial unless $\Sigma_{B}^{\mathrm{in}}=3 \mathbf{E}_{6}$, i.e., unless $B$ is as in Theorem 1.3.1(2).

Proof. The epimorphism part follows from Proposition 3.6.1, and the kernel of $\kappa$ is estimated using Corollaries 3.2.7 and 3.5.5.

3.6.3. Proposition. For a $\mathbb{D}_{6}$-sextic $B$ with $\Sigma_{B}^{\text {in }}=3 \mathbf{E}_{6}$, cf. Theorem 1.3.1(2), the representation $\bar{\pi}_{0}: \operatorname{Sym}_{\mathrm{st}}\left(\Gamma_{B}, \mathcal{K}_{B}\right) \rightarrow \mathbb{S}\left(\bar{\Gamma}_{B}\right)=\mathbb{S}_{3}$ is an isomorphism.

Proof. $\bar{\pi}_{0}$ is an epimorphism due to Proposition 3.6.1 and Corollary 3.5.5. Its kernel is trivial due to Corollary 3.2.11.

3.7. Sextics of weight eight. Let $B$ be a $\mathbb{D}_{6}$-sextic of weight 8 . According to Corollaries 3.2.6 and 3.5.4, the representation $\kappa: \operatorname{Sym}_{\mathrm{st}}\left(\Gamma_{B}, \mathcal{K}_{B}\right) \rightarrow G L\left(\mathcal{K}_{B}\right)$ as in (3.2.2) is monic. In [9], we constructed a stable symmetry $c$ of order 2 whose image in $G L\left(\mathcal{K}_{B}\right)$ is the central element - id; the minimal resolution of singularities of the quotient $\mathbb{P}^{2} / c$ is a geometrically ruled rational surface $\Sigma_{2}$ with an exceptional section $E$ of self-intersection (-2), and the image $B / c$ is a trigonal curve $\bar{B} \subset \Sigma_{2}$ with four cusps, $c f$. Section 4.2 and Theorem 4.2.1. The original plane $\mathbb{P}^{2}$ is the double covering of the quadratic cone $\Sigma_{2} / E$ ramified at the vertex $E / E$ and a certain section $\bar{L}$ of $\Sigma_{2}$, and $B$ is the pull-back of $\bar{B}$.

Since $c$ is central, any other stable symmetry of $B$ would descend to a symmetry of $\left(\Sigma_{2}, \bar{B}+\bar{L}\right)$ stable under equisingular deformations of $\bar{B}+\bar{L}$. The curve $\bar{B}$ is rigid; in appropriate affine coordinates $(x, y)$ in $\Sigma_{2}$ it is given by the polynomial

$$
f(x, y)=4 y^{3}-\left(24 x^{3}+3\right) y+\left(8 x^{6}+20 x^{3}-1\right) .
$$

The group of (Klein) symmetries of $B$ is the alternating group $\mathbb{A}_{4}$ (respectively, symmetric group $\mathbb{S}_{4}$ ); it can be identified with the group of even (respectively, all) permutations of the four cusps of $\bar{B}$, see [9]. (Recall that a Klein automorphism of an analytic variety is an either holomorphic or anti-holomorphic automorphism.) Since $B$ has no stable symmetries of order 3 , it suffices to show that $\bar{B}+\bar{L}$ has no stable symmetries of order 2. (Note that $\left|G L\left(\mathcal{K}_{B}\right)\right|=(3)(2)^{4}$.) All order 2 elements in $\mathbb{A}_{4}$ are conjugate, and one of them is given by the change of coordinates

$$
(x, y)=\left(-\frac{x^{\prime}-\epsilon}{2 \epsilon^{2} x^{\prime}+1},-\frac{3 y^{\prime}}{\left(2 \epsilon^{2} x^{\prime}+1\right)^{2}}\right), \quad \epsilon=-\frac{1}{2}+i \frac{\sqrt{3}}{2} .
$$

A section of $\Sigma_{2}$ is preserved by this transformation if and only if it has the form $y=a\left(2 x^{2}+2 \epsilon x-\epsilon^{2}\right), a \in \mathbb{C}$, and it is straightforward that the family $\bar{B}+\bar{L}$ with $\bar{L}$ as above does not contain any equisingular stratum; hence, $B$ has no other stable symmetries.

3.7.1. Remark. There is a unique section $\bar{L}=\{y=0\}$ that is invariant under the full group $\mathbb{S}_{4}$ of Klein symmetries of $\bar{B}$. It gives rise to a unique, up to projective transformation, sextic $B$ of weight 8 (with the set of singularities $8 \mathbf{A}_{2}$ ) for which the image of the representation $\bar{\kappa}$ : $\operatorname{Sym} B \rightarrow P G L\left(\mathcal{K}_{B}\right) \cong \mathbb{S}_{4}$, see (3.2.3), is the subgroup $\mathbb{A}_{4} \subset \mathbb{S}_{4}$. Using [9], this image can be identified with the group of even permutations of the four torus structures of $B$. 
3.8. Proof of Theorem 1.3.1. Proposition 3.1.1 states that most irreducible sextics admitting stable symmetries are $\mathbb{D}_{2 p}$-sextics, $p=3,5,7$. The exceptional case of sextics with two type $\mathbf{E}_{8}$ singular points is covered by Proposition 3.1.2.

The group of stable symmetries of $\mathbb{D}_{10^{-}}$and $\mathbb{D}_{14}$-sextics are found in Sections 3.3 and 3.4, respectively. $\mathbb{D}_{6}$-sextics of weight $w \leqslant 7$ are covered by Corollary 3.6.2 and Proposition 3.6.3, sextics of weight 8 are considered in Section 3.7, and the remaining case of sextics of weight 9 is contained in [9].

\section{Stable involutions}

In this section, we analyze the relation between stable involutions of irreducible sextics and maximal trigonal curves in $\Sigma_{2}$.

4.1. Maximal trigonal curves in $\Sigma_{2}$. The Hirzebruch surface $\Sigma_{k}, k \geqslant 0$, is a geometrically ruled rational surface with an exceptional section $E$ of square $-k$. A trigonal curve is a curve $B \subset \Sigma_{k}$ disjoint from $E$ and intersecting each generic fiber at three points. In appropriate affine coordinates $(x, y)$ in $\Sigma_{k}$, a trigonal curve can be given by its Weierstraß equation $y^{3}+g_{2}(x) y+g_{3}(x)=0$, where $\operatorname{deg} g_{2} \leqslant 2 k$ and $\operatorname{deg} g_{3} \leqslant 3 k$, and the (functional) $j$-invariant of $B$ is defined as the function

$$
j=j_{B}: \mathbb{P}^{1} \rightarrow \mathbb{P}^{1}, \quad x \mapsto \frac{4 g_{2}^{3}(x)}{\Delta(x)}, \quad \text { where } \Delta=4 g_{2}^{3}+27 g_{3}^{2} .
$$

Here, the first copy of $\mathbb{P}^{1}$ (the source) is the base of the ruling of $\Sigma_{k}$, and the second copy (the target) is the standard Riemann sphere $\mathbb{C} \cup \infty$. The curve $B$ is called isotrivial if $j_{B}=$ const.

By a singular fiber of a trigonal curve $B \subset \Sigma_{k}$ we mean a fiber of the ruling of $\Sigma_{k}$ intersecting $B$ geometrically at less than three points. Locally, in a neighborhood of a simple singular fiber, $B$ is the ramification locus of the Weierstraß model of a Jacobian elliptic surface, and to describe the type of the fiber we use (one of) the standard notation for the singular elliptic fibers, referring to the extended Dynkin graph of the exceptional divisors. For non-simple singular fibers, we use Arnol'd's notation $\tilde{\mathbf{J}}_{k, p}$ and $\tilde{\mathbf{E}}_{6 k+\epsilon}, k \geqslant 2$, referring to the type of the singular point of $B$.

The $j$-invariant has three special values, 0,1 , and $\infty$, which are typically taken at the roots of $g_{2}, g_{3}$, and $\Delta$, respectively. In [7], a trigonal curve $B$ with double singular points only is called maximal if $j_{B}$ has the following properties:

(1) $j_{B}$ has no critical values other than 0,1 , or $\infty$, and

(2) each pull-back $j_{B}^{-1}(0)$ (respectively, $j_{B}^{-1}(1)$ ) has ramification index at most three (respectively, at most two).

In order to extend this definition to all trigonal curves, we need to exclude singular fibers similar to $\tilde{\mathbf{D}}_{4}$, which are not detected by the $j$-invariant and typically increase the dimension of the moduli space. (Essentially, the additional requirement means that each singular fiber should remain singular after elementary transformations.) Thus, we have the following definition.

4.1.2. Definition. A trigonal curve $B \subset \Sigma_{k}$ is called maximal if $B$ has no singular fibers of type $\tilde{\mathbf{D}}_{4}$ or $\tilde{\mathbf{J}}_{k, 0}, k \geqslant 2$, and $j_{B}$ satisfies conditions (1), (2) above.

With this definition, the alternative characterization of maximal curves given in [7] still holds: a non-isotrivial curve $B$ is maximal if and only if it does not admit a nontrivial degeneration to another non-isotrivial trigonal curve. 
A singular fiber of $B$ is called stable if it is preserved by small equisingular (but not necessarily fiberwise) deformations of $B$. Clearly, stable are all fibers except those of type $\tilde{\mathbf{A}}_{0}^{* *}, \tilde{\mathbf{A}}_{1}^{*}$, or $\tilde{\mathbf{A}}_{2}^{*}$ (which can split into a stable fiber and $\tilde{\mathbf{A}}_{0}^{*}$ ). The curve $B$ is called stable if all its singular fibers are stable.

4.1.3. Theorem. Up to automorphism of $\Sigma_{2}$, a stable maximal trigonal curve $B \subset \Sigma_{2}$ is determined by its set of singular fibers, which can be one of those listed in Table 1 .

TABLE 1. Singular fibers of stable maximal curves in $\Sigma_{2}$

Irreducible curves

$$
\begin{array}{ll}
\tilde{\mathbf{E}}_{8} \oplus 2 \tilde{\mathbf{A}}_{0}^{*} & 2 \tilde{\mathbf{A}}_{4} \oplus 2 \tilde{\mathbf{A}}_{0}^{*} \\
\tilde{\mathbf{E}}_{6} \oplus \tilde{\mathbf{A}}_{2} \oplus \tilde{\mathbf{A}}_{0}^{*} & 4 \tilde{\mathbf{A}}_{2} \\
\tilde{\mathbf{A}}_{8} \oplus 3 \tilde{\mathbf{A}}_{0}^{*} &
\end{array}
$$

Reducible curves

$$
\begin{array}{ll}
\tilde{\mathbf{E}}_{7} \oplus \tilde{\mathbf{A}}_{1} \oplus \tilde{\mathbf{A}}_{0}^{*} & \tilde{\mathbf{A}}_{7} \oplus \tilde{\mathbf{A}}_{1} \oplus 2 \tilde{\mathbf{A}}_{0}^{*} \\
\tilde{\mathbf{D}}_{8} \oplus 2 \tilde{\mathbf{A}}_{0}^{*} & \tilde{\mathbf{A}}_{5} \oplus \tilde{\mathbf{A}}_{2} \oplus \tilde{\mathbf{A}}_{1} \oplus \tilde{\mathbf{A}}_{0}^{*} \\
\tilde{\mathbf{D}}_{6} \oplus 2 \tilde{\mathbf{A}}_{1} & 2 \tilde{\mathbf{A}}_{3} \oplus 2 \tilde{\mathbf{A}}_{1} \\
\tilde{\mathbf{D}}_{5} \oplus \tilde{\mathbf{A}}_{3} \oplus \tilde{\mathbf{A}}_{0}^{*} &
\end{array}
$$

4.1.4. Remark. In Table 1 , the curves with a type $\tilde{\mathbf{E}}$ singular fiber (and only these curves) admit equisingular isotrivial degenerations: $\tilde{\mathbf{E}}_{8} \oplus \tilde{\mathbf{A}}_{0}^{* *}, \tilde{\mathbf{E}}_{7} \oplus \tilde{\mathbf{A}}_{1}^{*}$, and $\tilde{\mathbf{E}}_{6} \oplus \tilde{\mathbf{A}}_{2}^{*}$.

Proof. According to [7], a maximal trigonal curve $B \subset \Sigma_{k}$ with double singular points only is determined, up to automorphism of $\Sigma_{k}$, by its skeleton $\operatorname{Sk} B \subset \mathbb{P}^{1} \cong$ $S^{2}$ (Grothendieck's dessin d'enfants), which is defined as the bi-partite planar map $j_{B}^{-1}([0,1])$, the pull-backs of 0 and 1 being, respectively, black and white vertices of $\mathrm{Sk} B$. The skeleton has the following properties:

(1) $\mathrm{Sk} B$ is connected;

(2) Sk $B$ has at least one black and at least one white vertex;

(3) the valency of each black (white) vertex is $\leqslant 3$ (respectively, $\leqslant 2$ ).

Conversely, ani bi-partite planar map satisfying (1)-(3) above is the skeleton of a certain maximal trigonal curve $B \subset \Sigma_{k}$; the parameter $k$ is given by the relation $2 k=b_{1}+2 b_{2}+b_{3}+w_{1}$, where $b_{i}$ and $w_{i}$ are the numbers of, respectively, black and white vertices of valency $i$.

Since the skeleton $\mathrm{Sk} B$ is a bi-partite graph, each complementary region of $\mathrm{Sk} B$ has equal numbers of black and white vertices ('corners') in the boundary; we call a region a $p$-gon, $p \geqslant 1$, if it has $p$ black and $p$ white corners. The stable singular fibers of $B$ are in a one-to-one correspondence with the regions of Sk $B$ : each $p$-gonal region contains a single singular fiber of type $\tilde{\mathbf{A}}_{p-1}\left(\tilde{\mathbf{A}}_{0}^{*}\right.$ if $\left.p=1\right)$. The unstable fibers of type $\tilde{\mathbf{A}}_{0}^{* *}, \tilde{\mathbf{A}}_{1}^{*}$, and $\tilde{\mathbf{A}}_{2}^{*}$ are over, respectively, the 1-valent black vertices, 1 -valent white vertices, and 2 -valent black vertices. For this reason, we call black vertices of valency $\leqslant 2$ and white vertices of valency 1 unstable.

Thus, in order to classify stable trigonal curves in $\Sigma_{2}$ with double singular points only, it suffices to list al bi-partite planar maps satisfying (1)-(3) above, with four trivalent black vertices, and without unstable vertices. This is done in Figure 1. (In the figures, we omit bivalent white vertices; one such vertex is to be placed at the center of each edge connecting two black vertices.) Reducible curves are detected using the criterion found in [7]. (It is worth mentioning that the skeleton is a graph in the oriented sphere $\mathbb{P}^{1}$. However, all graphs shown in Figures 1 and 2 are symmetric. In particular, this means that all curves are real.) 


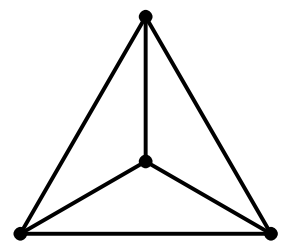

$4 \tilde{\mathbf{A}}_{2}$

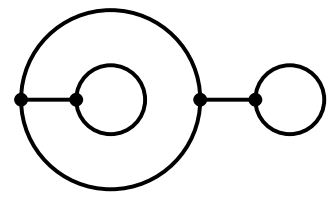

$2 \tilde{\mathbf{A}}_{4} \oplus 2 \tilde{\mathbf{A}}_{0}^{*}$

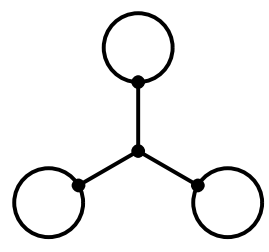

$\tilde{\mathbf{A}}_{8} \oplus 3 \tilde{\mathbf{A}}_{0}^{*}$

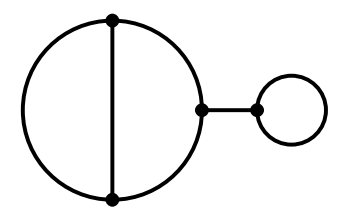

$\tilde{\mathbf{A}}_{5} \oplus \tilde{\mathbf{A}}_{2} \oplus \tilde{\mathbf{A}}_{1} \oplus \tilde{\mathbf{A}}_{0}^{*}$

FiguRE 1. Stable maximal curves in $\Sigma_{2}$ with double points only

If the curve has one simple triple point, we apply an elementary transformation centered at this point and obtain a maximal trigonal curve $B_{1} \subset \Sigma_{1}$ with at most one unstable fiber. Such curves are classified in Figure 2. If $B_{1}$ is stable, the inverse elementary transformation can contract any singular fiber of $B$, resulting in the curves with a type $\tilde{\mathbf{D}}$ singular fiber in Table 1 . If $B_{1}$ has an unstable singular fiver of type $\tilde{\mathbf{A}}_{0}^{* *}, \tilde{\mathbf{A}}_{1}^{*}$, or $\tilde{\mathbf{A}}_{2}^{*}$, the inverse elementary transformation should contract this fiber, resulting in a singular fiber of type $\tilde{\mathbf{E}}_{6}, \tilde{\mathbf{E}}_{7}$, or $\tilde{\mathbf{E}}_{8}$, respectively. To detect the reducible curves, one can either use the criterion in [7] or just notice that trigonal curves in $\Sigma_{1}$ are merely plane cubics.

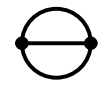

$3 \tilde{\mathbf{A}}_{1}$

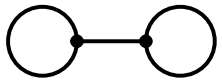

$\tilde{\mathbf{A}}_{3} \oplus 3 \tilde{\mathbf{A}}_{0}^{*}$

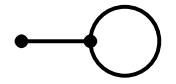

$\tilde{\mathbf{A}}_{0}^{* *} \oplus \tilde{\mathbf{A}}_{2} \oplus \tilde{\mathbf{A}}_{0}^{*}$

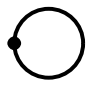

$\tilde{\mathbf{A}}_{2}^{*} \oplus 2 \tilde{\mathbf{A}}_{0}^{*}$

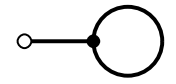

$$
\tilde{\mathbf{A}}_{1}^{*} \oplus \tilde{\mathbf{A}}_{1} \oplus \tilde{\mathbf{A}}_{0}^{*}
$$

Figure 2. Maximal curves in $\Sigma_{1}$ with at most one unstable fiber

To complete the proof, it remains to notice that a non-isotrivial trigonal curve in $\Sigma_{2}$ cannot have two triple points or a non-simple triple point (adjacent to $\mathbf{J}_{10}$ in Arnol'd's notation), as otherwise one would apply two elementary transformations and obtain a trigonal curve in $\Sigma_{0}$, which is necessarily isotrivial.

4.1.5. Corollary. Up to automorphism of $\Sigma_{2}$, a stable maximal trigonal curve $B \subset \Sigma_{2}$ is determined by its set of singularities.

Proof. The set of singularities of $B$ is obtained from its set of singular fibers by disregarding the type $\tilde{\mathbf{A}}_{0}^{*}$ summands and 'removing the tildes.' From Table 1 it follows that the two sets determine each other. Furthermore, the maximality of a set of singular fibers can be tested numerically, by applying the Riemann-Hurwitz formula to $j_{B}$. (For example, if all singular fibers are of type $\tilde{\mathbf{A}}_{p}, p \geqslant 1$, or $\tilde{\mathbf{A}}_{0}^{*}$, the curve is maximal if and only if the number of singular fibers is four.) Hence, each set of singularities obtained from Table 1 is realized by maximal curves (or their equisingular isotrivial degenerations, see Remark 4.1.4) only. 
4.1.6. Corollary. A non-isotrivial trigonal curve $B \subset \Sigma_{8}$ is stable and maximal if and only if $\mu(B)=8$.

Proof. The direct statement follows from Table 1. For the converse, we compare two independent classifications. A necessary condition for a set of simple singularities $\Sigma$ to be realized by a trigonal curve in $\Sigma_{2}$ is that $\Sigma$, regarded as a root system, must admit an embedding to $\mathbf{E}_{8}$, see, e.g., [6]. In addition to those listed in Table 1, there are three such root systems of rank eight: $2 \mathbf{D}_{4}, \mathbf{D}_{4} \oplus 4 \mathbf{A}_{1}$, and $8 \mathbf{A}_{1}$. The former set of singularities is realized by isotrivial curves (obtained by two elementary transformations from a union of three disjoint sections of $\Sigma_{0}$ ). The sets of singularities $\mathbf{D}_{4} \oplus 4 \mathbf{A}_{1}$ and $8 \mathbf{A}_{1}$ are not realized by a trigonal curve in $\Sigma_{2}$ : each singular fiber of type $\tilde{\mathbf{D}}_{4}$ (respectively, $\tilde{\mathbf{A}}_{1}$ ) is a root of the discriminant $\Delta$, see (4.1.1), of multiplicity 6 (respectively, 2 ), whereas $\operatorname{deg} \Delta \leqslant 12$.

4.1.7. Remark. As it follows from the proof of Corollary 4.1.6, the isotrivial curves $B \subset \Sigma_{2}$ with $\mu(B)=8$ are either the equisingular isotrivial degenerations listed in Remark 4.1.4 or the curve with the set of singular fibers $2 \tilde{\mathbf{D}}_{4}$.

4.2. Stable involutions. Recall that the set of fixed points of an involutive automorphism $c$ of $\mathbb{P}^{2}$ consists of a line $L_{c}$ and an isolated point $O_{c}$. The blown up quotient $\mathbb{P}^{2}\left(O_{c}\right) / c$ is the Hirzebruch surface $\Sigma_{2}$; the exceptional divisor over $O_{c}$ projects to the exceptional section $E \subset \Sigma_{2}$, and the line $L_{c}$ projects to a generic section $\bar{L} \subset \Sigma_{2}$ disjoint from $E$. Conversely, given a section $\bar{L} \subset \Sigma_{2}$ disjoint from $E$, the double covering of $\Sigma_{2} / E$ ramified at $\bar{L}$ and $E / E$ is the plane $\mathbb{P}^{2}$, and the deck translation of the covering is an involutive automorphism whose fixed point set is the pull-back of the union $\bar{L} \cup(E / E)$.

4.2.1. Theorem. Let $B \subset \mathbb{P}^{2}$ be an irreducible sextic with simple singularities, and let $c \in \operatorname{Sym}_{\mathrm{st}} B$ be an involutive stable symmetry of $B$. Then the image of $B$ in the Hirzebruch surface $\Sigma_{2}=\mathbb{P}^{2}\left(O_{c}\right) / c$ is an irreducible stable maximal trigonal curve $\bar{B}$ (or an equisingular isotrivial degeneration of such a curve, see Remark 4.1.4). The set of singularities of $\bar{B}$ is as follows:

(1) $\mathbf{E}_{8}$, if $B$ is as in 1.3.1(8);

(2) $\mathbf{E}_{6} \oplus \mathbf{A}_{2}$, if $B$ is as in 1.3.1(2) or (4);

(3) $\mathbf{A}_{8}$, if $B$ is as in 1.3.1(5);

(4) $2 \mathbf{A}_{4}$, if $B$ is as in 1.3.1(7);

(5) $4 \mathbf{A}_{2}$, if $B$ is as in 1.3.1(1) or (6).

Proof. According to [8], the image $\bar{B}$ is either a trigonal curve or a hyperelliptic curve with $\bar{B} \circ E=2$; in both cases, the singularities of $\bar{B}$ can be found using the results of [8]. Assuming that $\bar{B}$ is a trigonal curve, the essential singular points of $B$ project to the sets of singularities listed in the statement, while the ordinary singular points give rise to points of tangency of $\bar{B}$ and $\bar{L}$. To complete the proof in this case, one applies Corollary 4.1.6.

It remains to rule out the possibility that $\bar{B}$ is a hyperelliptic curve. As, in this case, $\bar{B}$ cannot have a triple point, it cannot appear from a sextic $B$ as in 1.3.1(2), (4), or (8). The sextics as in 1.3.1(1), (6) and 1.3.1(7) were treated in [9] and [8], respectively. The only remaining possibility is the set of essential singularities $\mathbf{A}_{17}$ in 1.3.1(5), which can be obtained from a hyperelliptic curve $\bar{B}$ with a single type $\mathbf{A}_{7}$ singular point on $E$. Such a curve $\bar{B}$ does exist, but it is necessarily reducible (see, e.g., [3]); hence, so is $B$. 
4.2.2. Theorem. An involutive symmetry $c$ of an irreducible plane sextic $B$ with simple singularities is stable if and only if the image of $B$ in the Hirzebruch surface $\Sigma_{2}=\mathbb{P}^{2}\left(O_{c}\right) / c$ is an irreducible stable maximal trigonal curve (or an equisingular isotrivial degeneration of such a curve, see Remark 4.1.4).

Proof. The 'only if' part is given by Theorem 4.2.1. The 'if' part follows essentially from comparing Theorems 4.2.1 and 4.1.3: in addition, one needs to check that, for each degeneration of the section $\bar{L}$ (passing through a singular point of $\bar{B}$, tangency to $\bar{B}$, etc.), the dimension of the moduli space of such sections coincides with its expected dimension, which in turn equals the dimension of the moduli space of corresponding sextics, $c f$. Remark 4.2 .5 below. We leave details to the reader. (In fact, the sets of singularities $2 \mathbf{A}_{4}$ and $4 \mathbf{A}_{2}$ were studied in [8] and [9], respectively; the three other sets of singularities will be considered in a subsequent paper.)

4.2.3. Conjecture. An involutive symmetry $c$ of a plane sextic $B$ with simple singularities only is stable if and only if the image of $B$ in the Hirzebruch surface $\Sigma_{2}=\mathbb{P}^{2}\left(O_{c}\right) / c$ is a stable maximal trigonal curve (or an equisingular isotrivial degeneration of such a curve, see Remark 4.1.4).

4.2.4. Remark. A simple parameter count shows that, for sextics with a nonsimple singular point, the conclusion of Conjecture 4.2.3 fails. For example, some sextics $B$ with the sets of singularities $\mathbf{Y}_{1,1}^{1} \oplus 2 \mathbf{A}_{4}, \mathbf{Y}_{1,1}^{1} \oplus \mathbf{A}_{9}$, and $\mathbf{W}_{12} \oplus 2 \mathbf{A}_{4}$ admit a symmetry $c$ such that the image of $B$ in $\mathbb{P}^{2}\left(O_{c}\right) / c$ is the maximal trigonal curve with the set of singularities $2 \mathbf{A}_{4}$. However, such sextics form codimension 1 subsets in their equisingular strata, see [8].

4.2.5. Remark. One can use a similar parameter count to substantiate the 'if' part of Conjecture 4.2.3. From the description of the moduli of sextics, see [4], it follows that, for any sextic $B$ with simple singularities, $\operatorname{dim} \mathcal{M}_{6}\left(\Sigma_{B}\right)=19-\mu(B)$. Let $B$ be the double covering of a stable maximal trigonal curve $\bar{B} \subset \Sigma_{2}$ ramified at $E$ and a section $\bar{L}$. If $\bar{L}$ is transversal to $\bar{B}$, then $\mu(B)=2 \mu(\bar{B})=16$, see Corollary 4.1.6, and $\operatorname{dim} \mathcal{M}_{6}\left(\Sigma_{B}\right)=3$ is the dimension of the space of sections of $\Sigma_{2}$. Each constraint on $\bar{L}$ (passing though a singular point of $\bar{B}$, tangency to $\bar{B}$, etc.) increases $\mu(B)$ by one while decreasing by one the dimension of the space of sections (assuming that the constraints are independent). Thus, in all cases, the dimensions of the moduli spaces coincide, i.e., the deck translation of the covering is a symmetry of each generic curve in $\mathcal{M}_{6}\left(\Sigma_{B}\right)$.

\section{REFERENCES}

1. A. Beauville, Application aux espaces de modules, Géométrie des surfaces K3: modules et périodes, Astérisque, vol. 126, 1985, pp. 141-152.

2. N. Bourbaki, Groupes et algèbres de Lie, Chapitres 4, 5 et 6, Masson, 1981.

3. A. Degtyarev, Isotopy classification of complex plane projective curves of degree 5, Algebra i Analis 1 (1989), no. 4, 78-101 (Russian); English transl. in Leningrad Math. J. 1 (1990), no. $4,881-904$.

4. A. Degtyarev, On deformations of singular plane sextics, J. Algebraic Geom. 17 (2008), $101-135$.

5. A. Degtyarev, Oka's conjecture on irreducible plane sextics, arXiv:math.AG/0701671.

6. A. Degtyarev, Oka's conjecture on irreducible plane sextics. II, arXiv:math.AG/0702546.

7. A. Degtyarev, Zariski k-plets via dessins d'enfants, arXiv:0710.0279.

8. A. Degtyarev, On irreducible sextics with non-abelian fundamental group, arXiv:0711.3070.

9. A. Degtyarev, Irreducible plane sextics with large fundamental groups, arXiv:0712.2290.

10. A. Degtyarev, M. Oka, A plane sextic with finite fundamental group, arXiv:0711.3067. 
11. V. V. Nikulin, Integer quadratic forms and some of their geometrical applications, Izv. Akad. Nauk SSSR, Ser. Mat 43 (1979), 111-177 (Russian); English transl. in Math. USSR-Izv. 43 (1980), 103-167.

12. V. V. Nikulin, Finite groups of automorphisms of Kählerian K3-surfaces, Trudy Moskov. Mat. Obshch. 38 (1979), 75-137 (Russian); English transl. in Trans. Moscow Math. Soc. (1980), no. 2, 71-135.

13. M. Oka, D. T. Pho, Classification of sextics of torus type, Tokyo J. Math. 25 (2002), no. 2, 399-433.

14. A. Özgüner, Classical Zariski pairs with nodes, M.Sc. thesis, Bilkent University, 2007.

Department of Mathematics, Bilkent University, 06800 Ankara, Turkey

E-mail address: degt@fen.bilkent.edu.tr 